\title{
Watching National Team Matches in World Cup Soccer 2014 on Television was Associated with Increasing Frequency of Premature Ventricular Contractions
}

\author{
Tomoyuki Shiozawa $^{1}$, Kazunori Shimada ${ }^{1 *}$, Gaku Sekita ${ }^{1}$, Hidemori Hayashi ${ }^{1}$, Haruna Tabuchi ${ }^{1}$, Sayaka \\ Komatsu$^{1}$, Tetsuro Miyazaki', Katsumi Miyauchi ${ }^{2}$, Jun Shitara ${ }^{3}$, Takuma Yoshihara ${ }^{2}$, Eiryu Sai ${ }^{2}$, Mas- \\ ayuki Shiozaki ${ }^{4}$, Kosuke Fukao ${ }^{5}$, Yoshifumi Fukushima ${ }^{6}$, Shinichiro Yamagami ${ }^{7}$, Satoru Suwa ${ }^{3}$, Youichi \\ Katoh $^{1}$, Yasumasa Fujiwara ${ }^{4}$, Hiroshi Ikeda ${ }^{8}$, Masataka Sumiyoshi ${ }^{4}$, Yuji Nakazato ${ }^{9}$, Hiroyuki Daida ${ }^{1}$ \\ ${ }^{1}$ Department of Cardiovascular Medicine, Juntendo University Graduate School of Medicine, Bunkyo-ku, Tokyo, Japan \\ ${ }^{2}$ Department of Cardiology, Juntendo Tokyo Koto Geriatric Medical Center, Koto-ku, Tokyo, Japan \\ ${ }^{3}$ Department of Cardiology, Juntendo University, Shizuoka Hospital, Izunokuni-shi, Shizuoka, Japan \\ ${ }^{4}$ Department of Cardiology, Juntendo University, Nerima Hospital, Nerima-ku, Tokyo, Japan \\ ${ }^{5}$ Department of Sports Medicine, Juntendo University, Faculty of Health and Sports Science, Inzai, Chiba, Japan \\ ${ }^{6}$ Department of Cardiology, Koshigaya Municipal Hospital, Koshigaya, Saitama, Japan \\ ${ }^{7}$ Department of Cardiology, Kyoundo Hospital, Bunkyo-ku, Tokyo, Japan \\ ${ }^{8}$ Department of Orthopedic Surgery, Juntendo University, Graduate School of Medicine, Bunkyo-ku, Tokyo, Japan \\ ${ }^{9}$ Department of Cardiology, Juntendo University, Urayasu Hospital, Urayasu, Chiba, Japan
}

Corresponding author: Kazunori Shimada, MD, Department of Cardiovascular Medicine, Juntendo University, Graduate School of Medicine, 2-1-1 Hongo, Bunkyo-ku, Tokyo-113-8421, Japan, E-mail: shimakaz@juntendo.ac.jp

\begin{abstract}
Objective: Psychological triggers, such as emotional stress, increase the incidence of acute cardiovascular events. The association between soccer championships and risk of cardiovascular events remains controversial. A World Cup Soccer (WCS) match involving a national team might be a strong enough trigger to induce cardiovascular events. However, there are no reports of a multicenter study that has investigated the relationship between watching WCS and cardiac arrhythmia.

Methods: We assessed 25 patients who were evaluated for ischemic changes and/or arrhythmia using 24-h Holter electrocardiography in four cardiology divisions during WCS 2014. The patients were divided into two groups: the watching (W) group consisted of 7 patients who watched WCS on live television and the Non-Watching (NW) group consisted of 18 patients who did not watch WCS. Heart rates, arrhythmia, and ischemic changes were evaluated.

Results: There were no differences in the clinical characteristics, heart rates, premature atrial contraction frequencies, and ischemic changes between the two groups. Although there were no differences in total Premature Ventricular Contractions (PVCs), the frequency of PVCs during matches $(61 \pm 101$ vs. $7 \pm 8, \mathrm{P}=0.03)$ and 1 hour before matches $(15 \pm 17$ vs. $3 \pm 5, \mathrm{P}=0.01)$ were significantly higher in the $\mathrm{W}$ group than in the NW group. No sustained ventricular tachycardia or fibrillation was observed.

Conclusions: A significant association between watching WCS and frequency of PVCs was observed in patients with/or suspected of having cardiovascular disease.
\end{abstract}

Received Date: February 13, 2016 Accepted Date: March 28, 2016 Published Date: April 01, 2016

Citation: Shimada, K., et al. Watching National Team Matches in World Cup Soccer 2014 on Television was Associated with Increasing Frequency of Premature Ventricular Contractions. (2016) J Heart Cardiol 2(1): 17-21.

DOI: $10.15436 / 2378-6914.16 .022$

\section{Introduction}

Emotional stress is well known as a risk factor for cardiovascular events ${ }^{[1-3]}$. However, the risk of cardiovascular events caused by watching soccer games remains controversial. Until now, there has been no multicenter study that has investigated the relationship between watching soccer games and cardiac arrhythmia evaluated by 24-hours Holter Electrocardiography (Holter ECG). World Cup Soccer (WCS) was held in Brazil from June to July 2014. In this study, we investigated the frequency of arrhythmia and ischemic changes using Holter ECG in patients with cardiovascular diseases and those suspected of having cardiac disease during the Japan National Team's matches in WCS 2014. 


\section{Materials and Methods}

Study population: We enrolled 25 patients (mean age $63 \pm 19$ years, 10 males) who underwent Holter ECG in the outpatient clinic of four cardiology divisions on June 19 and June 24, 2014. During the WCS qualifying rounds, Japan versus Greece and Japan versus Columbia were broadcast on live television at 7 AM on June 20 and 5 AM on June 25, 2014 (Japan time). The patients were not informed about the study protocol before Holter ECG but were notified about the study when they returned the Holter ECG recorders during which they were asked if they had watched any soccer matches on live television. Then, the patients were divided into two groups: the watching (W) group consisted of 7 patients who had watched WCS on live television and the non-watching (NW) group consisted of 18 subjects who had not watched WCS. All protocols were approved by the ethics committee of Juntendo University (14-032), and all participants provided an informed consent before their participation in this study according to the guidelines established in the Declaration of Helsinki.

Measurements: We measured the total number of heart beats, frequency of premature atrial contractions (PACs) and Premature Ventricular Contractions (PVCs), and ischemic changes using a 24-hours Holter ECG system (SCM-8000; FUKUDA DENSHI, Tokyo, Japan). These events were measured each hour. Ischemic changes were also diagnosed using ST-segment depression in the CM5 lead.

\section{Statistical analysis}

Continuous variables were compared using the unpaired t-test or Mann-Whitney U-test, and categorical variables were compared using the chi-squared test. A value of $\mathrm{P}<0.05$ was considered to indicate statistical significance. All data were analyzed using JMP® 11 for Windows (SAS Institute Inc., Cary, NC, USA).

\section{Results}

Patient characteristics: The clinical characteristics of the study subjects are shown in Table 1 . Seventeen patients (61\%) had cardiovascular diseases (ten patients with a trial fibrillation, three with coronary artery disease, four with valvular heart disease, three with cerebrovascular disease, and one with hypertrophic cardiomyopathy). The clinical characteristics, including age, sex, body mass index, coronary risk factors, and underlying disease, did not differ between the groups. The concomitant use of medications in each group is shown in Table 2. There were no significant differences in medical treatment between the groups.

Table 1: Patient Characteristics

\begin{tabular}{|c|c|c|c|}
\hline & $\begin{array}{c}\text { Watching group } \\
\quad(n=7)\end{array}$ & $\begin{array}{c}\text { Non-watching group } \\
(\mathrm{n}=18)\end{array}$ & p value \\
\hline Age, years & $65 \pm 16$ & $62 \pm 20$ & 0.74 \\
\hline Male gender, \% & $3(43)$ & $7(39)$ & 0.86 \\
\hline Body mass index, $\mathrm{kg} / \mathrm{m}^{2}$ & $23.3 \pm 3.7$ & $21.4 \pm 2.9$ & 0.17 \\
\hline Hypertension, \% & $4(57)$ & $7(39)$ & 0.41 \\
\hline Dyslipidemia, \% & $3(43)$ & $7(39)$ & 0.86 \\
\hline Diabetes mellitus, \% & $2(29)$ & $2(11)$ & 0.29 \\
\hline Current smoker, \% & $0(0)$ & $1(6)$ & 0.52 \\
\hline Coronary artery disease, $\%$ & $1(14)$ & $2(11)$ & 0.83 \\
\hline Valvular heart disease, \% & $1(10)$ & $3(17)$ & 0.88 \\
\hline Hypertrophic cardiomyopathy, \% & $0(0)$ & $1(6)$ & 0.52 \\
\hline Prior stroke, $\%$ & $1(14)$ & $2(11)$ & 0.83 \\
\hline Atrial fibrillation, $\%$ & $3(43)$ & $7(39)$ & 0.86 \\
\hline CHADS2 score & $1.6 \pm 1.4$ & $1.0 \pm 1.2$ & 0.31 \\
\hline LVEF, \% & $61.8 \pm 6.3$ & $65.3 \pm 7.3$ & 0.34 \\
\hline E/e' & $17.8 \pm 20.3$ & $10.9 \pm 5.5$ & 0.22 \\
\hline \multicolumn{4}{|c|}{$\begin{array}{l}\text { Unless otherwise indicated, data are presented as mean } \pm \text { SD. } \\
\text { CHADS } 2 \text { score is a measure of the risk of stroke (in which congestive heart failure, hypertension, an age of } 75 \text { years or older, } \\
\text { and diabetes mellitus are each assigned } 1 \text { point, and previous stroke or transient ischemic attack is assigned } 2 \text { points; the score is } \\
\text { calculated by summing all the points for a given patient stroke risk); E/e', mitral inflow E/E' ratio determined by Doppler echocar- } \\
\text { diogram is an indicator of left ventricular diastolic function; LVEF, left ventricular ejection fraction. }\end{array}$} \\
\hline
\end{tabular}


Table 2: Concomitant use of medications

\begin{tabular}{|l|c|c|c|}
\hline & $\begin{array}{c}\text { Watching group } \\
(\mathbf{n}=\mathbf{7})\end{array}$ & $\begin{array}{c}\text { Non-watching group } \\
(\mathbf{n}=\mathbf{1 8})\end{array}$ & p value \\
\hline Aspirin, \% & $1(14)$ & $1(6)$ & 0.47 \\
\hline Dual antiplatelets, \% & $1(14)$ & $0(0)$ & 0.10 \\
\hline Anticoagulant, \% & $2(29)$ & $6(33)$ & 0.82 \\
\hline Diuretics, \% & $1(14)$ & $3(17)$ & 0.88 \\
\hline ACE-I/ARB, \% & $3(43)$ & $3(17)$ & 0.17 \\
\hline $\boldsymbol{\beta}$-blocker, \% & $2(29)$ & $7(39)$ & 0.63 \\
\hline Statin, \% & $2(29)$ & $3(17)$ & 0.50 \\
\hline ACE-I - angiotensin-converting enzyme inhibitor; ARB - angiotensin- $\beta$ receptor blocker & \\
\hline
\end{tabular}

Arrhythmic and ischemic events: A comparison of Holter ECG data between the groups is shown in Table 3. There was no significant difference in the mean heart rate for 24 hours between the groups. There was also no significant difference in total PVCs over 24 hours. However, the frequency of PVCs during the $2 \mathrm{~h}$ of each match was significantly higher in the W group than that in the NW group (61 \pm 101 vs. $7 \pm 8, \mathrm{P}=0.03$ ) (Figure). The frequency of PVCs $1 \mathrm{~h}$ before matches was also significantly higher in the $\mathrm{W}$ group than that in the NW group $(15 \pm 17$ vs. $3 \pm 5, \mathrm{P}=0.01)$. There was no significant difference in the frequency of PVCs 1 hour after matches. There was no significant difference in total PACs during the matches between the groups. There were also no significant differences in the levels of maximum ST depression over the 24 hours and during the 2 hours of matches between the groups. No ventricular tachycardia, ventricular fibrillation, or other cardiovascular events occurred during the study period.

Table 3: Comparison of Holter ECG data between the two groups

\begin{tabular}{|c|c|c|c|}
\hline & $\begin{array}{l}\text { Watching group } \\
\quad(\mathrm{n}=7)\end{array}$ & $\begin{array}{l}\text { Non-watching group } \\
\qquad(\mathrm{n}=18)\end{array}$ & p value \\
\hline Total heart beats, beats & $95059 \pm 10821$ & $102004 \pm 15550$ & 0.29 \\
\hline Mean heart rate, bpm & $73 \pm 18$ & $75 \pm 14$ & 0.78 \\
\hline Heart rate during match $(2 \mathrm{~h}), \mathrm{bpm}$ & $82 \pm 18$ & $76 \pm 12$ & 0.29 \\
\hline \multicolumn{4}{|l|}{ PACs, beats } \\
\hline Total PACs & $5220 \pm 10672$ & $1298 \pm 2698$ & 0.16 \\
\hline PACs during match (2h) & $411 \pm 770$ & $117 \pm 268$ & 0.17 \\
\hline \multicolumn{4}{|l|}{ PVCs, beats } \\
\hline Total PVCs & $672 \pm 845$ & $237 \pm 472$ & 0.11 \\
\hline$-2 \mathrm{~h} \sim-1 \mathrm{~h}$ before match & $6 \pm 13$ & $3 \pm 7$ & 0.43 \\
\hline$-1 \mathrm{~h} \sim 0 \mathrm{~h}$ before match & $15 \pm 17$ & $3 \pm 5$ & 0.01 \\
\hline $0 \mathrm{~h} \sim 1 \mathrm{~h}$ of match & $15 \pm 21$ & $2 \pm 2$ & 0.01 \\
\hline $1 \mathrm{~h} \sim 2 \mathrm{~h}$ of match & $46 \pm 84$ & $4.8 \pm 7$ & 0.052 \\
\hline $0 \mathrm{~h} \sim+1 \mathrm{~h}$ after match & $30 \pm 34$ & $22 \pm 69$ & 0.78 \\
\hline$+1 \mathrm{~h} \sim+2 \mathrm{~h}$ after match & $21 \pm 28$ & $19 \pm 45$ & 0.95 \\
\hline PVCs 2h during match & $61 \pm 101$ & $7 \pm 8$ & 0.03 \\
\hline PVCs in $22 \mathrm{~h}$ except match & $611 \pm 758$ & $230 \pm 471$ & 0.14 \\
\hline PVCs $2 \mathrm{~h}$ before match & $21 \pm 29$ & $6 \pm 10$ & 0.06 \\
\hline PVCs $2 h$ after match & $48 \pm 58$ & $38 \pm 106$ & 0.83 \\
\hline PVCs/Total heart beats, \% & $0.74 \pm 0.96$ & $0.2 \pm 0.5$ & 0.10 \\
\hline PVCs 2h during match/total PVCs, \% & $7.1 \pm 7.1$ & $6.0 \pm 7.2$ & 0.73 \\
\hline PVCs $2 \mathrm{~h}$ during match/PVC in other $22 \mathrm{~h}, \%$ & $8.3 \pm 8.8$ & $7.1 \pm 9.2$ & 0.78 \\
\hline CM5 maximum ST depression, $\mathrm{mV}$ & $-1.08 \pm 1.52$ & $-0.58 \pm 0.84$ & 0.30 \\
\hline CM5 max ST depression during match, mV & $-0.39 \pm 0.41$ & $-0.38 \pm 0.82$ & 0.99 \\
\hline
\end{tabular}




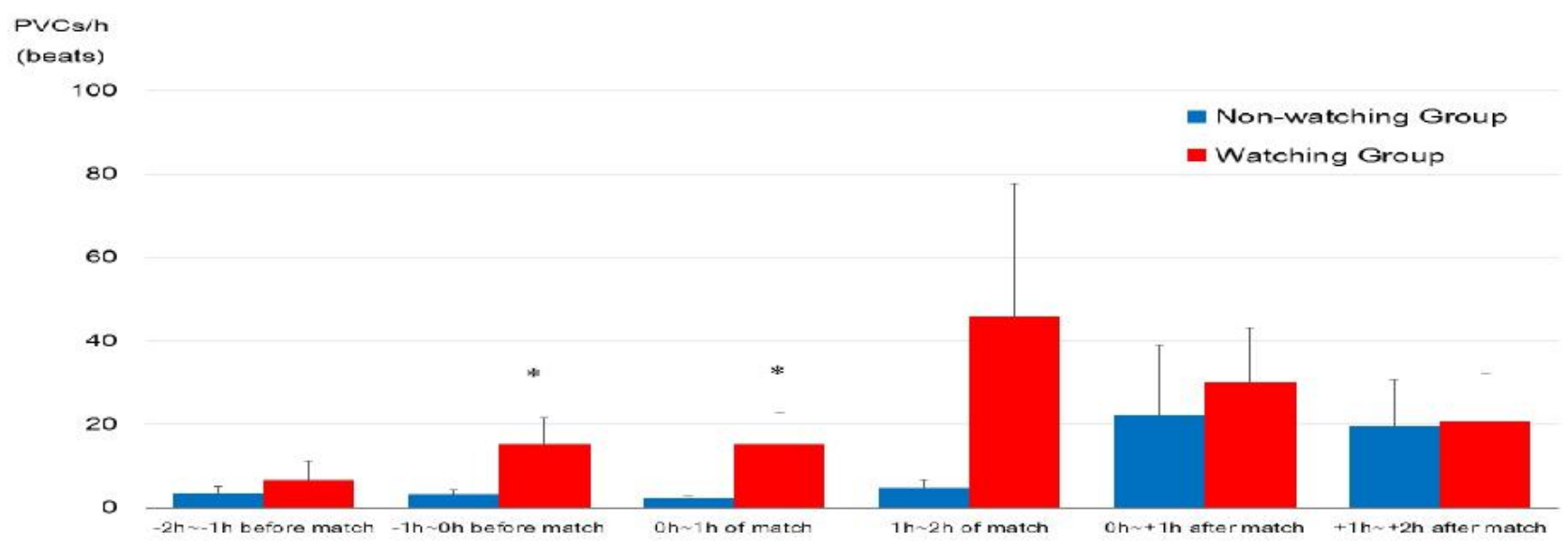

Figure1: PVCs relative to the start of the match.

$\mathrm{h}$; hours. Data are represented as the mean \pm standard error. * $\mathrm{p}<0.05$

\section{Discussion}

This study demonstrated a significant association between watching WCS and frequency of PVCs in patients with cardiovascular diseases and patients suspected of having cardiovascular disease. To the best of our knowledge, this is the first multicenter study to investigate the prevalence of arrhythmia evaluated by Holter ECG in patients with/or suspected of having cardiovascular diseases who watched WCS.

Previous studies have shown that at least $20 \%$ of episodes of life-threatening ventricular arrhythmia and unexpected death occurred under the influence of acute emotional stress ${ }^{[3-7]}$. Acute and chronic emotional stress has been shown to induce autonomic nervous system activation and provoke physical disabilitie ${ }^{[8]}$. These strong mental stressors influence the sympathetic tone, increase blood pressure and vasoconstriction, produce inflammatory mediators, cause endothelial dysfunction, and lead to a hypercoagulable state, which can trigger cardiovascular events. It has been suggested that increases in heart rate, blood pressure, myocardial contractility, coronary flow, and coronary tone may promote vulnerable plaque rupture ${ }^{[9]}$. Studies have shown that disasters such as earthquakes increase the occurrences of acute coronary syndromes, and ventricular tachyarrhythmia and hospitalization in patients with implantable cardiac defibrillators ${ }^{[10]}$. Also the prevalence of post-traumatic stress disorder after earthquakes, increased the frequency of death, acute myocardial infarction, stroke, and heart failure ${ }^{[11]}$.

Watching a soccer match is often associated with emotional stress. Fédération Internationale de Football Association (FIFA) WCS involving national teams is one of the most popular sporting events in the world. In the WCS in 1998, England and Argentina played each other in the first round of the finals, and Argentina won in a penalty shootout. The Hospital Episode Statistics in England showed an increase in hospital admission for acute myocardial infarctions during the following 3 days relative to those on other days without matches. The increase in myocardial infarctions could have been triggered by emotional distress, such as a favorite football team losing in an important match ${ }^{[12]}$. When France, as the host country, won a game in the WCS, cardiovascular mortality in men decreased on the game day, which suggested that the immense fervor and collective euphoria observed at the time of a victory could decrease mortality ${ }^{[13]}$. In the WCS 2006, Germany lost in overtime in the semi-finals. The prevalence of cardiac emergencies was 2.66 times higher on the days of matches than during the control period. In patients with coronary artery disease, the prevalence of emergencies was 4.03 times higher than that during the control period. The highest number of events was observed within the 2 hours of the match. The next highest risk periods was each 2 hours before and after the match ${ }^{[14]}$. These data suggested that watching a stressful soccer match was a risk for acute cardiovascular events. In addition, a significant increase in PVCs during a match and 1 hour before a match is consistent with the results of the present study. As discussed above, several studies have reported the increase in cardiovascular emergencies during the events ${ }^{[15-19]}$. However, a recent study has reported that watching soccer was not necessarily associated with an increased incidence of cardiovascular events ${ }^{[20]}$. These data suggest that further studies are needed to clarify our findings.

The Japan National Football Team had a scoreless draw and lost in the next match. In this study, the matches were in the early morning on June 20 (7 AM kickoff, Japan time) and June 25 (5 AM kickoff, Japan time). The wake-up time may have affected the study results because these factors may be associated with sympathetic tone, less sleep, and more drinking of alcohol and caffeine, in early morning matches ${ }^{[2]}$ However, only four patients slept during the two matches in the NW group. No significant differences in the total PVCs, PVCs during matches, or PVCs in other hours were shown between the awaking subjects and sleeping subjects in the NW group (data not shown).

In this study, nonsustained ventricular tachycardia or fatal arrhythmia was not observed. It has been reported that prediction of cardiac and arrhythmogenic death was strongly associated with the prevalence of PVCs and nonsustained ventricular tachycardia that was a trigger of sustained ventricular tachycardia and ventricular fibrillation ${ }^{[22]}$. The prevalence of high risk patients, such as those with ischemic heart disease, was relatively low, and patients with ongoing chronic heart failure were not included in this study. 
Most of the patients underwent Holter ECG to evaluate the cause of palpitation and chest pain and/or were examined to determine the number of extra heart beats. For these reasons, ventricular tachycardia and fatal arrhythmia were not observed in this study.

This study had several limitations. First, this was a retrospective multicenter study with a small sample size. Prospective studies with a large number of subjects are needed to confirm our findings. Second, as mentioned above, the reason for performing Holter ECG was mainly to evaluate palpitation, chest pain, and extra systole. Therefore, the present results might not be representative of other subjects. Third, heart rate variability and heart rate turbulence were not evaluated. Fourth, alcohol and caffeine intake were not quantified, which might be confounding factors. Finally, all subjects were Japanese. Racial differences in enthusiasm during soccer matches may have been a major limitation.

\section{Conclusion}

In conclusion, a significant association between watching WCS and frequency of PVCs was observed in patients with cardiovascular diseases and patients who were suspected of having cardiac diseases.

Funding: This work was supported by the Ministry of Education, Culture, Sports, Science, and Technology-supported Program for the Strategic Research Foundation at Private Universities, Japan.

Conflict of interest: There were no conflicts of interest.

\section{References}

1. Schwartz, B.G., French, W.J., Mayeda, G.S., et al. Emotional stressors trigger cardiovascular events. (2012) Int J Clin Pract 66(7): 631-639.

2. Esler, M., Kaye, D. Sympathetic nervous system activation in essential hypertension, cardiac failure and psychosomatic heart disease. (2000) J Cardiovasc Pharmacol 35(7 Suppl 4): S1-S7.

3. Reich, P., DeSilva, R.A., Lown, B., et al. Acute psychological disturbances preceding life-threatening ventricular arrhythmias. (1981) JAMA 246(3): 233-235.

4. Ziegelstein, R.C. Acute emotional stress and cardiac arrhythmias. (2007) JAMA 298(3): 324-329.

5. Lampert, R., Jain, D., Burg, M.M., et al. Destabilizing effects of mental stress on ventricular arrhythmias in patients with implantable cardioverter-defibrillators. (2000) Circulation 101(2): 158-164.

6. Grippo, A.J., Johnson, A.K. Stress, depression and cardiovascular dysregulation: a review of neurobiological mechanisms and the integration of research from preclinical disease models. (2009) Stress 12(1): 1-21.

7. Kop, W.J., Weissman, N.J., Zhu, J., et al. Effects of acute mental stress and exercise on inflammatory markers in patients with coronary artery disease and healthy controls. (2008) Am J Cardiol 101(6): 767-773.
8. Terranova, C., Snenghi, R., Thiene, G., et al. Psychic trauma as cause of death. (2011) Med Sci Law 51(Suppl 1): S11-S15.

9. Falk, E. Why do plaques rupture? (1992) Circulation $86(6$ Suppl): III30-III42.

10. Nakano, M., Kondo, M., Wakayama, Y., et al. Increased incidence of tachyarrhythmias and heart failure hospitalization in patients with implanted cardiac devices after the great East Japan earthquake disaster. (2012) Circ J 76(5): 1283-1285.

11. Onose, T., Nochioka, K., Sakata, Y., et al. Predictors and prognostic impact of post-traumatic stress disorder after the great East Japan earthquake in patients with cardiovascular disease. (2015) Circ J 79(3): 664-647.

12. Carroll, D., Ebrahim, S., Tilling, K., et al. Admissions for myocardial infarction and World Cup football: database survey. (2002) BMJ 325(7378): 1439-1442.

13. Berthier, F., Boulay, F. Lower myocardial infarction mortality in French men the day France won the 1998 World Cup of football. (2003) Heart 89(5): 555-556.

14. Wilbert-Lampen, U., Leistner, D., Greven, S., et al. Cardiovascular Events during World Cup Soccer. (2008) N Engl J Med 358(5):475-483.

15. Katz, E., Metzger, J.T., Marazzi, A., et al. Increase of sudden cardiac deaths in Switzerland during the 2002 FIFA World Cup. (2006) Int J Cardiol 107(1): 132-133.

16. Katz, E., Metzger, J.T., Schlaepfer, J., et al. Increase of out-of-hospital cardiac arrests in the male population of the French speaking provinces of Switzerland during the 1998 FIFA World Cup. (2005) Heart 91(8): 1096-1097.

17. Kirkup, W., Merrick, D.W. A matter of life and death: population mortality and football results. (2003) J Epidemiol Community Health 57(6): 429-432.

18. Witte, D.R., Bots, M.L., Hoes, A.W., et al. Cardiovascular mortality in Dutch men during 1996 European football championship: longitudinal population study. (2000) BMJ 321(7276): 1552-1524.

19. Borges, D.G., Monteiro, R.A., Schmidt, A., et al. World soccer cup as a trigger of cardiovascular events. (2013) Arq Bras Cardiol 100(6): 546-552.

20. Niederseer, D., Thaler, C.W., Egger, A., et al. Watching soccer is not associated with an increase in cardiac events. (2013) Int J Cardiol 170(2): 189-194.

21. Willich, S.N. Circadian variation and triggering of cardiovascular events. (1999) Vasc Med 4(1): 41-49.

22. Scirica, B.M., Braunwald, E., Belardinelli, L., et al. Relationship between nonsustained ventricular tachycardia after non-ST-elevation acute coronary syndrome and sudden cardiac death: observations from the metabolic efficiency with ranolazine for less ischemia in non-ST-elevation acute coronary syndrome-thrombolysis in myocardial infarction 36 (MERLIN-TIMI 36) randomized controlled trial. (2010) Circulation 122(5): 455-462.
Journal ISSN: 2378-6914 (online)

Journal Title: Journal of Heart and Cardiology

Short title : J Heart Cardiol

www.ommegaonline.org
Ommega Online Publishers

E-mail: cardiology@ommegaonline.com

Website: www.ommegaonline.org 